\title{
Modeling Chamber Transport for Heavy-Ion Fusion
}

W.M. Sharp, D.A. Callahan-Miller, M. Tabak, S.S. Yu, P.F. Peterson, D.R. Welch, D.V. Rose

This article was submitted to: $2^{\text {nd }}$ IAEA Technical Meeting on Physics and Technology of Inertial Fusion Energy

Targets and Chambers, San Diego, CA, June 17-19, 2002

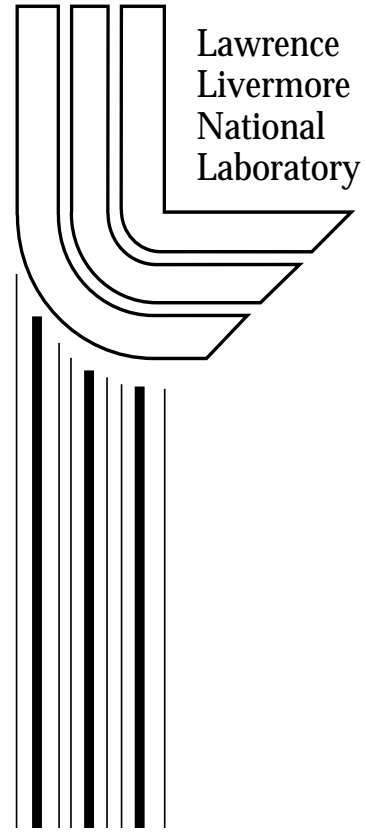

August 2, 2002 


\section{DISCLAIMER}

This document was prepared as an account of work sponsored by an agency of the United States Government. Neither the United States Government nor the University of California nor any of their employees, makes any warranty, express or implied, or assumes any legal liability or responsibility for the accuracy, completeness, or usefulness of any information, apparatus, product, or process disclosed, or represents that its use would not infringe privately owned rights. Reference herein to any specific commercial product, process, or service by trade name, trademark, manufacturer, or otherwise, does not necessarily constitute or imply its endorsement, recommendation, or favoring by the United States Government or the University of California. The views and opinions of authors expressed herein do not necessarily state or reflect those of the United States Government or the University of California, and shall not be used for advertising or product endorsement purposes.

This is a preprint of a paper intended for publication in a journal or proceedings. Since changes may be made before publication, this preprint is made available with the understanding that it will not be cited or reproduced without the permission of the author. 


\title{
Modeling Chamber Transport for Heavy-Ion Fusion
}

\author{
W. M. Sharp, D. A. Callahan, M. Tabak \\ Lawrence Livermore National Laboratory, Livermore, CA 94550, USA \\ S. S. $\mathrm{Yu}$ \\ Lawrence Berkeley National Laboratory, Berkeley, CA 94720, USA \\ P. F. Peterson \\ University of California Berkeley, Berkeley, CA 95720, USA \\ D. R. Welch and D. V. Rose \\ Mission Research Corporation, Albuquerque, NM 87104, USA \\ C. L. Olson \\ Sandia National Laboratory, Albuquerque, NM 87107, USA
}

\begin{abstract}
In a typical thick-liquid-wall scenario for heavy-ion fusion (HIF), between seventy and two hundred high-current beams enter the target chamber through ports and propagate about three meters to the target. Since molten-salt jets are planned to protect the chamber wall, the beams move through vapor from the jets, and collisions between beam ions and this background gas both strip the ions and ionize the gas molecules. Radiation from the preheated target causes further beam stripping and gas ionization. Due to this stripping, beams for heavy-ion fusion are expected to require substantial neutralization in a target chamber. Much recent research has, therefore, focused on beam neutralization by electron sources that were neglected in earlier simulations, including emission from walls and the target, photoionization by the target radiation, and pre-neutralization by a plasma generated along the beam path. When these effects are included in simulations with practicable beam and chamber parameters, the resulting focal spot is approximately the size required by a distributed radiator target.
\end{abstract}

\section{INTRODUCTION}

Heavy-ion beams for an inertial-fusion driver will likely require some form of neutralization during their final transport to the target. The present generation of indirect-drive targets requires a total particle current exceeding $40 \mathrm{kA}$ divided between perhaps a hundred beams, and each beam must have focal spot on the target of about $2 \mathrm{~mm}$. In conceptual chamber designs with thick-liquid inner walls, this final transport distance is typically several meters, because it must house neutron shielding, two meters of molten-salt jets inside the chamber to cushion the detonation, and an additional meter of standoff so the jets do not vaporize. Focusing is further complicated by collisions between the beam and vapor from the molten salt, which increase the charge state of beam ions. Although several chamber-transport methods[1] have been proposed for heavy-ion fusion (HIF), the mode currently favored in the US program is "neutralized ballistic" transport. With this method, electrons from collisional ionization or from some external source are entrained by the beam and neutralize the space charge sufficiently that the pulse focuses on the target in a nearly ballistic manner.

In recent years, considerable progress has been made in numerical simulations of neutralized ballistic transport. A useful survey in 1995 by Callahan[2] indicated that beam stripping due to collisions with the background gas would complicate focusing when thick-liquid walls were used to protect the chamber. Much better focusing was found when as little as $0.44 \%$ of the background gas was ionized, although uniform ionization even at that low level remains problematic. Both Barboza[3] and Vay and Deutsch[4] reported improvements in numerics for chamber-transport simulations, and some of these advances were incorporated in subsequent work by Sharp, et al. [5]. None of this later work, however, changed the original conclusion by Callahan that supplemental neutralization is necessary for successful transport in a chamber protected by thick-liquid walls. In Japan, Kikuchi, et al.[6] have proposed using a dielectric liner in the beam ports to furnish electrons, while the US HIF program is studying the use of injected hydrogen plasma to pre-neutralize beams[7]. Recent simulations of foot pulses by Sharp, et al.[8] show that passing a beam through a low-density plasma substantially improves the focal spot, provided that the background-gas density is sufficiently low. In this work, however, the plasma is electrical isolated, reducing its effectiveness. New simulations by Rose, et al.[9] and by Welch, et al.[10] show that placing the plasma in contact with conducting walls leads to beam neutralization approaching the theoretical maximum and, consequently, to focal spots of $2 \mathrm{~mm}$ or less.

Most of the published work on neutralized chamber transport uses beam and chamber parameters near those chosen 


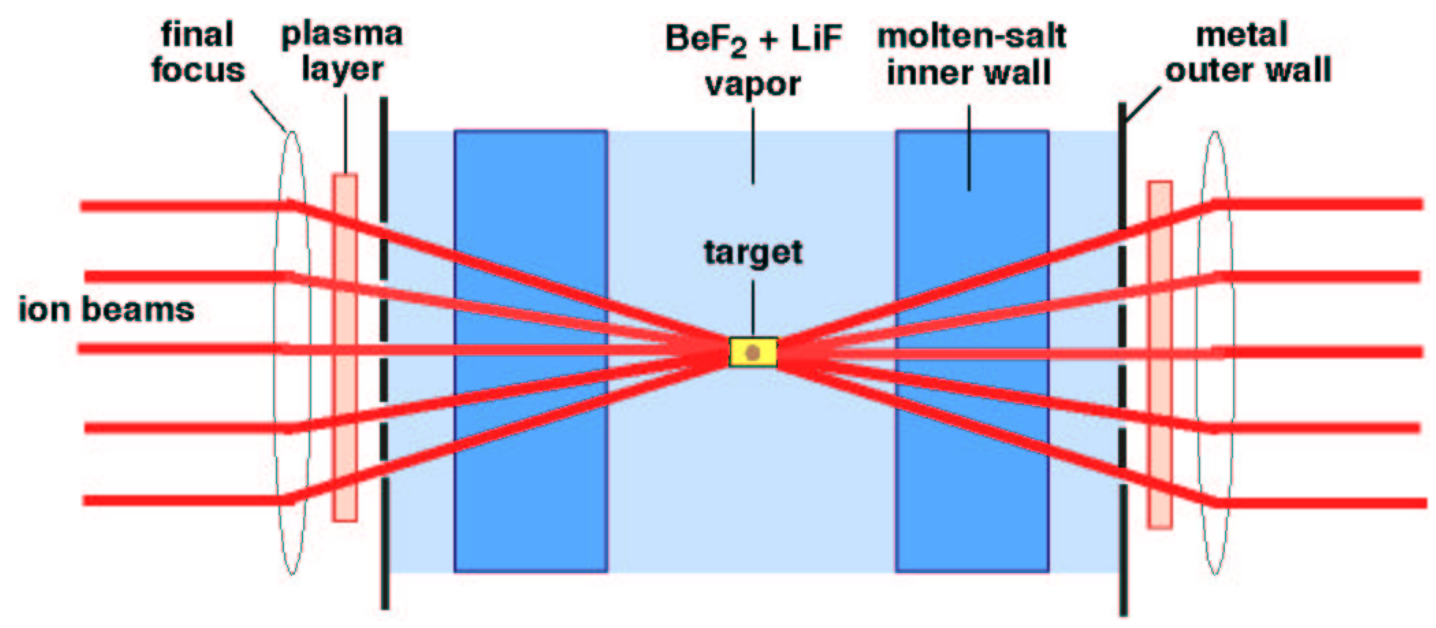

Figure 1: Sketch of a generic fusion chamber with an indirect-drive target driven on two sides by clusters of ion beams. The thick-liquid walls protecting the chamber, shown as solid shaded areas, would actually consist of crisscrossed jets of molten salt.

by Callahan[2], even though conceptual designs of HIF power plants now favor using lighter ions at lower energy and substantially higher current. The simulations in this paper are the first to use beam parameters and a chamber layout derived from recent power-plant studies. These parameters are presented in Section II, along with details of the numerical model. In Section III, we show that these beams marginally meet the requirements of current target designs. A brief summary and some comments about remaining work are given in a concluding section.

\section{METHOD}

\section{A. Numerical Model}

The axisymmetric chamber-transport simulations reported here were made with the electromagnetic particle-in-cell (PIC) code LSP [11, 12], developed by Mission Research Corporation. The code has a relativistic particle advance, allows the use of multiple species, and incorporates models of most physical processes expected in a fusion chamber, particularly electron emission from walls and collisional ionization and scattering. In addition, there are several features that make LSP useful for modeling chamber transport. The problem domain is built from simple geometric forms, allowing the beam port and chamber to be modeled together. The number of macroparticles representing any species can be controlled using an algorithm developed by Brackbill and Lapenta[13], so acceptable particle statistics can be maintained despite large differences in the densities of the various species. LSP has an optional implicit integration step, allowing the use of small grid cells when needed without being constrained by a Courant condition. Finally, a rudimentary photoionization model[5] has added to LSP for the present work to assess the effects of X rays emitted by the heated target.

The LSP chamber-transport model necessarily includes a wide range of physical processes. A complete representation of the fusion chamber, sketched in Fig. 1, would include neutralization by one or more upstream plasma layers, electron emission from walls, collisional scattering, ionization, and recombination between the beam and background gas, charge build-up on the target, modification of electric fields by the molten-salt jets, and photoionization of the beam, jets, and background gas by $\mathrm{X}$ rays from the heated target. The simulations presented here include the most important of these effects, although some, such as collisional scattering and recombination, are estimated to be small and are therefore neglected. Only a single beam is treated, ignoring possible inter-beam effects near the target, and the initial beam distribution function is idealized. The beam is assumed to be axisymmetric, with initially uniform density and emittance, and the current is constant except in rise and fall sections near the beam ends. The background gas in the chamber, resulting from the molten-salt jets, is assumed to be initially uniform and neutral, and it falls off with a physically plausible profile in the beam port. Due to the low background-gas density in the chamber, we follow collisional ionization only of neutral gas molecules, although the gas can be ionized to higher charge states by $\mathrm{X}$ rays from the target. Background-gas ions are mobile, whereas the hydrogen ions in the neutralizing plasma are not. Runs made with the opposite choices indicate, however, that ion mobility has a negligible effect. Finally, the 
molten-salt jets themselves are neglected at present, and there is no metal boundary where the target would be, so any problems with target-charge buildup would not appear.

The collisional-ionization cross sections used here were calculated by R. E. Olson[14] using a Monte-Carlo technique. Electrons liberated by collisions are assigned a Maxwellian energy distribution with an average energy of $20 \mathrm{eV}$, approximating the distribution calculated by Olson, and their angular distribution is random. In the absence of experimental validation, the Olson cross sections have instead been compared with results from a phenomenological model developed by Armel[15]. This comparison shows good agreement for beam ions with a charge state less than four, and discrepancies seen for higher charge states are not expected to compromise the results significantly, due to the small size of these higher-state cross sections. At present, the LSP model only allows single-electron ionization events, even though calculations[14] suggest that cross sections for multiple-electron ionization are sizeable. Nonetheless, multiple-electron ionization has been studied elsewhere[8] and is found to have only a minor effect on the neutralization and focal spot of a typical HIF beam.

\section{B. Parameters}

The simulation parameters used here are based on recent HIF power-plant studies by Meier[16]. The design code IBEAM $[17,18]$ minimizes the power-plant cost by adjusting parameters in an elaborate set of physics and pricing relationships. This optimization procedure typically involves tradeoffs that complicate chamber transport. Lowerenergy beams with higher current are favored because the cost of induction accelerators increases in rough proportion with ion energy. However, the lower energy necessitates use of a lower ion mass $M$ to give the same stopping distance in a target. As discussed in Ref. [8], the current per beam $I$ increases like $M^{-1}$ when the number of beams and their duration are held constant, and the generalized perveance[19], which is a good measure of space-charge effects, varies like $M^{-2}$ under these conditions. The higher total current needed with lighter ions can be partially mitigated by dividing the current between more beams, but a practical limit is set by achievable packing densities around the chamber, by the maximum angle at which beams can impinge on the target, and by overall system complexity. To increase thermal efficiency, the optimization model also favors a higher temperature for the molten-salt jets, although that choice increases the background-gas pressure and therefore the rate of collisional stripping. Finally, the IBEAM optimization is based on the requirements of distributed-radiator targets[20, 21], developed at Lawrence Livermore National Laboratory. Unlike previous target designs in which the ends of a cylindrical hohlraum were fully illuminated, this family of targets requires $95 \%$ of the beam energy to be deposited in an annulus on each end with a width between 1 and $2.3 \mathrm{~mm}$. Since the space-charge field of a beam scales inversely with the radius, the use of distributed-radiator targets at least doubles the maximum space-charge force of an unneutralized beam and quadruples the peak charge density.

The indirect-drive target described in Ref. [20] requires a total beam energy of $6-6.5$ MJ. About 1.5 MJ of this total must be delivered in a "foot" pulse, beginning $30 \mathrm{~ns}$ before the main pulses arrive, heating the hohlraum to about $100 \mathrm{eV}$ and initiating the first shock wave in the capsule. The main pulse then deposit their energy in 8-10 ns, launching three more shock waves and igniting the fuel. To compensate for range-shortening as the target is heated, the energy of foot-pulse ions is $75 \%$ of the main-pulse ion energy. Following Meier[16], we use $\mathrm{Xe}^{+1}$ ions at $131 \mathrm{amu}$ in the simulations here, with ion energies of $1.9 \mathrm{GeV}$ and $2.5 \mathrm{GeV}$ respectively in the foot and main pulses. Foot and main-pulse currents taken to be $0.76 \mathrm{kA}$ and $2.84 \mathrm{kA}$ respectively, corresponding to a total 112 beams, with 36 foot beams and 76 main beams. The main-pulse current profile has an 8-ns flat top, with 3 -ns parabolically varying rise and fall sections at the ends, while the foot-pulse profile has an 18-ns flat top and 6-ns rise and fall sections. Each type of beam has a 6 - $\mathrm{cm}$ radius as it enters the 3 - $\mathrm{m}$ beam port, and the beam is focused at a point $3 \mathrm{~m}$ inside the chamber wall, giving the beam a 6 - $\mathrm{m}$ transport distance and an initial convergence angle of $10 \mathrm{mrad}$. The unnormalized rms emittance $\epsilon_{\perp}$ is taken to be initially $9 \mathrm{~mm}$-mrad, but results are not sensitive to this choice, due the sizable emittance growth during chamber transport.

The chamber simulated here is uniformly filled with a mixture of $90 \% \mathrm{BeF}_{2}$ and $10 \% \mathrm{LiF}$, as expected when molten $\mathrm{FLiBe}[22]$ at $600^{\circ} \mathrm{C}$ is used to protect the chamber wall. Based experimental work by Olander, et al.[23], the vapor density in the chamber is $7 \times 10^{12} \mathrm{~cm}^{-3}$, corresponding to about $0.2 \mathrm{mTorr}$. In the 3 -m beam port, the density falls off proportionally with $\left(1+\Delta z^{2} / R_{\text {port }}^{2}\right)^{-1}$, where $R_{\text {port }}$ is the beam-port radius and $\Delta z$ is the distance upstream from the chamber. A $10-\mathrm{cm}$ layer of fully ionized hydrogen plasma is placed near each end of the beam port, one centered $36 \mathrm{~cm}$ from the entrance and the other, $12 \mathrm{~cm}$ from the exit. The electron density in the plasma is $3 \times 10^{11} \mathrm{~cm}^{-3}$, although simulation results are not sensitive to this density so long as it exceeds the beam density at the same axial position. The conducting beam-port wall is a cone placed 1-cm outside the nominal beam edge, and electron emission is allowed near the plasma layers. 

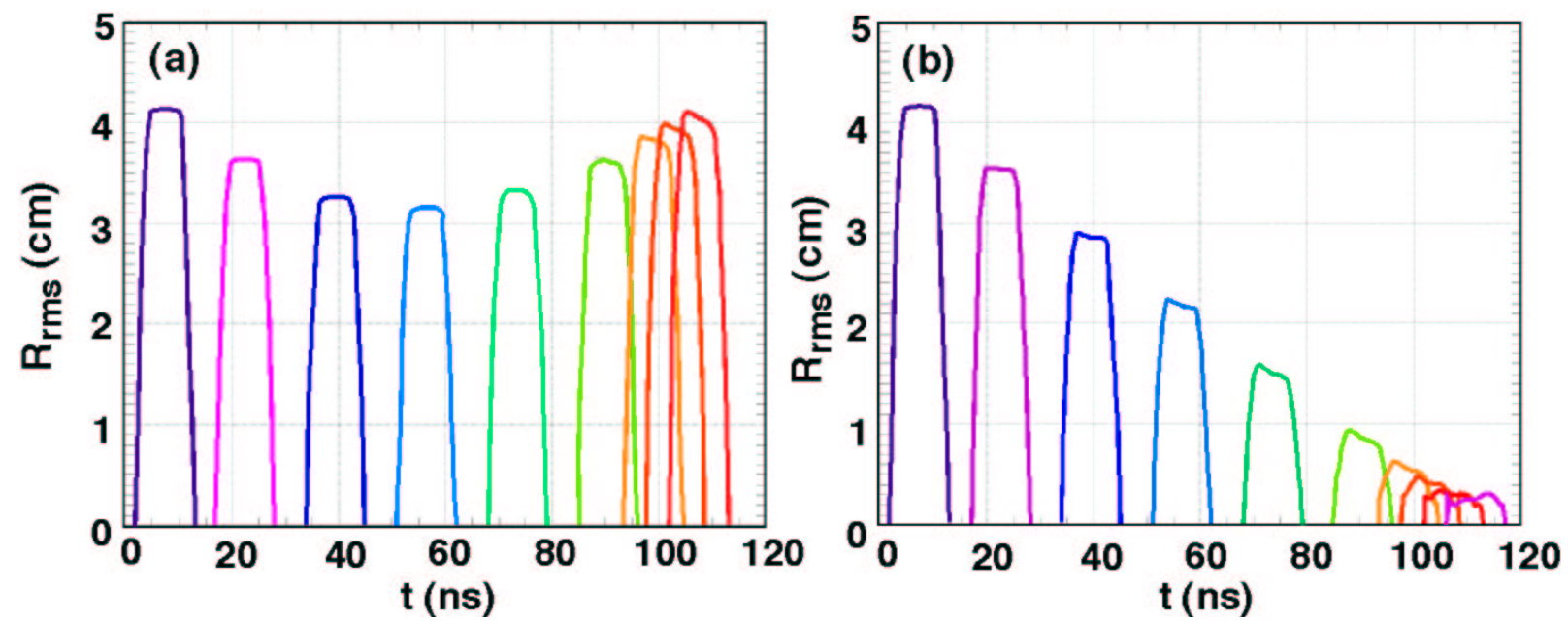

Figure 2: Time variation of the main-pulse rms radi at selected axial locations (a) with no pre-neutralization and (b) with pre-neutralizing plasma place nears the ends of the beam port. Photoionization has been turned off in these simulations.

\section{RESULTS}

\section{A. Effects of pre-neutralization}

To illustrate the effects of the pre-neutralization alone, we artificially turn off photoionization and compare the dynamics of beams with and without the upstream plasma layers. As found in earlier chamber-transport simulations[2], the neutralization provided by background-gas ionization for the present parameters is inadequate to allow a usable focal spot. Time histories of the beam rms radius for an unneutralized main pulse, shown in Fig. 2a, indicate that the point of best focus, called the beam "waist", occurs just as the beam enters the chamber, after 50 ns transport. By the time the beam reaches the nominal target location, about $100 \mathrm{~ns}$, the radius is larger than the initial value. When the case is rerun with the addition of plasma layers near the ends of the beam port, approximately $90 \%$ percent of the beam space charge is neutralized as it enters the chamber, and the minimum radius is about $2.6 \mathrm{~mm}$, as seen in Fig. 3b. The waist actually occurs $0.12 \mathrm{~m}$ beyond the nominal target location, due to the residual space charge, but this error can be corrected by changing the nominal focal position. Results with foot pulses are similar, with the radius at the waist location remaining less than $2.4 \mathrm{~mm}$ when pre-neutralization is used.

Another series of runs has shown that several factors contribute to the effectiveness of pre-neutralization. When the same beam is passed through electrically isolated plasmas, the emerging beam is only about $50 \%$ charge neutralized, since removal of electrons from the plasma builds up a space charge that resists further extraction. Surrounding the beam with a conducting but non-emitting pipe as it passes through the plasma increases the neutralization to nearly $80 \%$, because the image charge on the pipe alters the plasma space-charge field and makes it easier to remove electrons along the axis. Finally, permitting electron emission from the conducting wall keeps the plasma quasi-neutral as electrons are extracted, giving roughly $90 \%$ neutralization. This value is close to the theoretical limit for these parameters of about $95 \%$, calculated using work by C. L. Olson[1].

The effectiveness of pre-neutralization is found to decrease at higher background-gas densities. At the nominal density of $7 \times 10^{12} \mathrm{~cm}^{-3}$, the mean-free-path for background-gas ionization is $4.5 \mathrm{~m}$, which is substantially larger than the 3 -m chamber radius. Consequently, neither beam stripping nor background-gas ionization have much effect on beam neutralization. The average charge state of the beam remains less than two, and in the absence of the upstream plasma layers, neutralization from the background climbs only to about $70 \%$. In contrast, when the ionization length is short compared with the chamber radius, neutralization fraction is scarcely different that that from pre-neutralization alone, since that value is already near the theoretical limit, but the beam is stripped to a much higher charge state. As a result, ions feel a larger space-charge force and focus to a larger spot. 

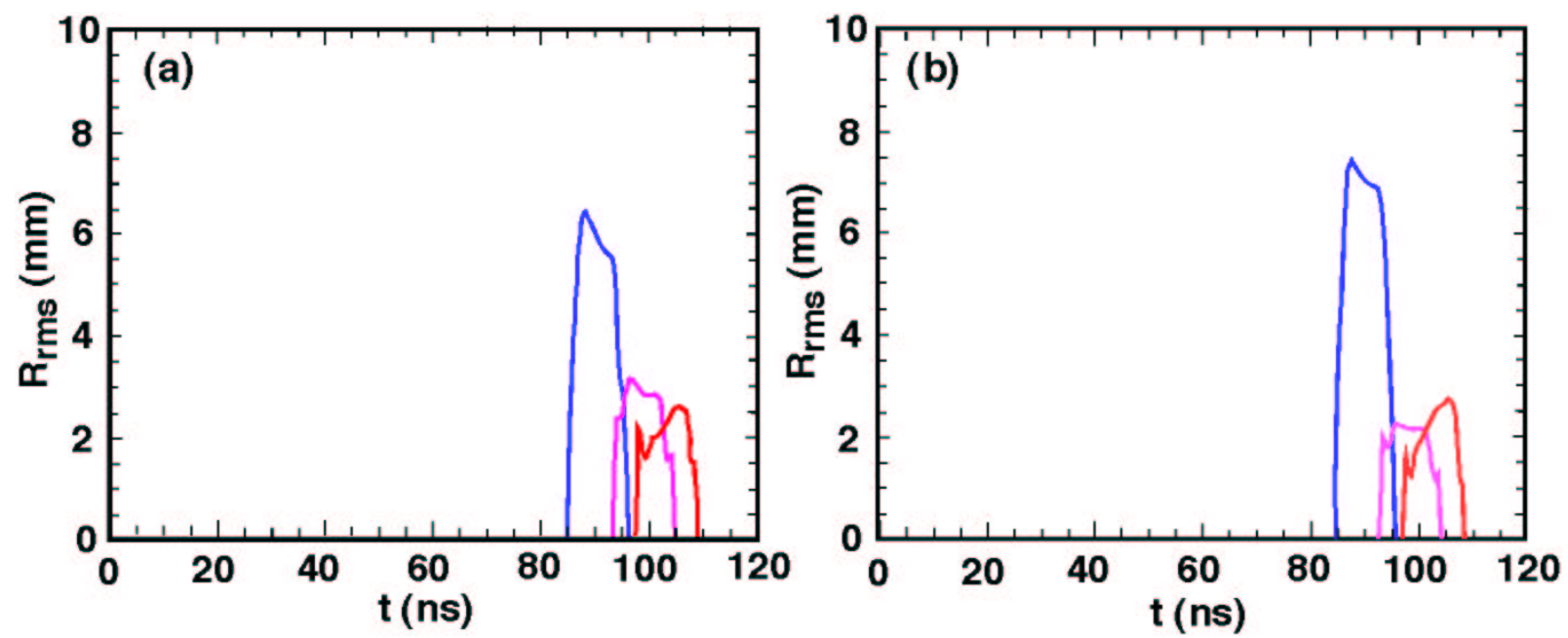

Figure 3: Time variation of the main-pulse rms radius with (a) a $10 \mathrm{mrad}$ and (b) a 15 mrad initial convergence angle. Photoionization has been turned off in these simulations.

\section{B. Effects of beam convergence angle}

One proposal for reducing the beam focal spot is to increase the initial convergence angle $\theta_{0}$. In the absence of space charge, an envelope equation for the beam rms radius[24] predicts a minimum radius of $\epsilon_{\perp} / \theta_{0}$, provided that the rms emittance $\epsilon_{\perp}$ is constant. LSP simulations using both the nominal 10-mrad convergence angle and a 15-mrad angle, again with photoionization turned off, show the waist radius varying more weakly with $\theta_{0}$ than the predicted inverse dependence. The time histories of the main-pulse radius in Fig. 3 show the minimum radius decreasing from $2.6 \mathrm{~mm}$ for $10 \mathrm{mrad}$ to $2.2 \mathrm{~mm}$ for $15 \mathrm{mrad}$, which is less than half the predicted $33 \%$ decrease. Also, the waist radius for both angles is about a factor of three larger than predicted by the envelope model due to the emittance increase during transport. Similar results are found for foot pulses. The maximum radius of a foot pulse decreases from $2.4 \mathrm{~mm}$ at $10 \mathrm{mrad}$ to $1.9 \mathrm{~mm}$ at $15 \mathrm{mrad}$, again less than the predicted change. In all these simulations, the analytic expression predicts too small a waist because it ignores emittance growth during transport, which is roughly a factor of three for the present parameters. In addition, the expression overestimates the sensitivity to the convergence angle because the beam space charge is imperfectly neutralized, contrary to what was assumed. The unneutralized space charge contributes an additional force that gives a finite waist even for zero emittance.

Although these simulations show some improvement from using a larger convergence angle, there are also several drawbacks. First, the larger beam size in the final-focus magnets would increase the size and cost of that section. Second, the larger entrance holes and gaps between molten-salt jets would allow more neutrons from a fusion target to escape the chamber, complicating the shielding problem. Finally, enlarging each beam line would increase the cone angle of beams approaching the target, forcing the use of a target with lower gain. The cone angle might be held constant by reducing the number of beams, but the current of each beam would then have to be increased appropriately, probably nullifying the improved focal spot. Selecting the optimum convergence angle will require collaboration with target designers and neutronics engineers.

\section{Effects of photoionization}

Including photoionization by target $\mathrm{X}$ rays in the previous case with a 15-mrad convergence angle makes only a modest improvement in chamber transport. Due to the $r^{-2}$ fall-off of the photon density, the effects of photoionization become significant only in the final $50-75 \mathrm{~cm}$ of transport. One major effect is the increase in the average beam charge state from about 1.8 to more nearly six, as shown in Fig. 4. This increase, however, has little effect on beam dynamics for two reasons. First, the beam is quite rigid. To bend a $2.5-\mathrm{GeV} \mathrm{Xe} \mathrm{Xe}^{+5}$ ion by $1 \mathrm{~mm}$ over a 50 -cm distance requires about $8.5 \mathrm{MV} / \mathrm{m}$, a value far exceeding the calculated net field. The second reason for the minor effect on dynamics is that the beam net charge increases relatively little in this region, due both to the abundance of free electrons from background-gas photoionization and to the fact that free electrons from photostripping are nearly co-moving with 

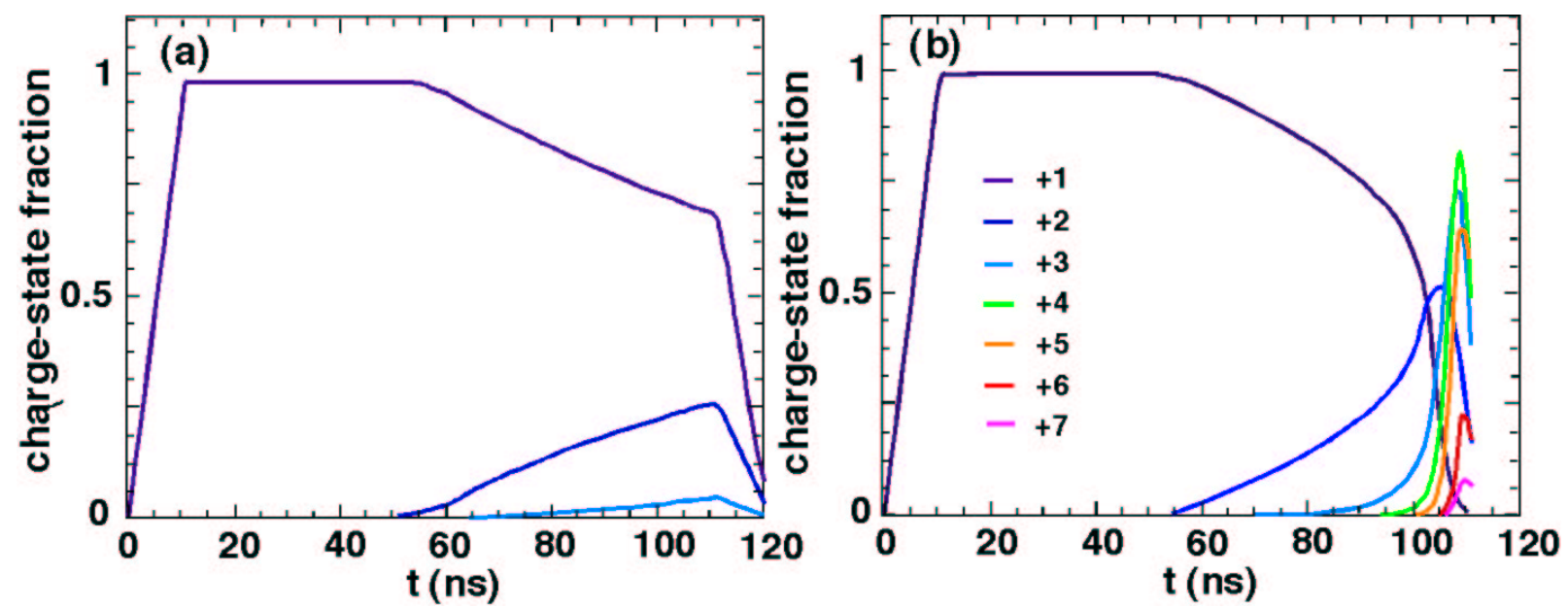

Figure 4: Time histories of the fraction of main-pulse ions in various charge states (a) with and (b) without photoionization. The beam head reaches the target location after about $100 \mathrm{~ns}$.
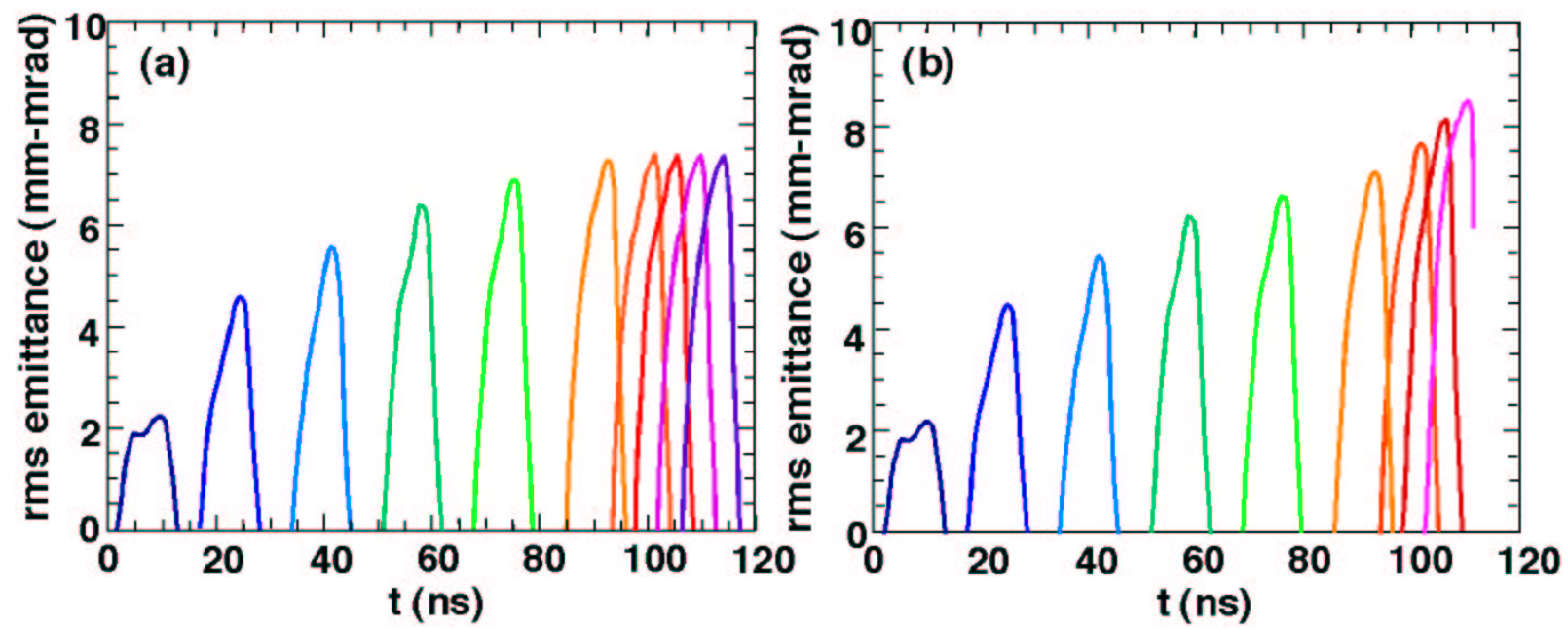

Figure 5: Time variation of the main-pulse emittance at selected axial positions (a) with and (b) without photoionization.

the parent ion. At the beam waist, the net charge inside the beam increases by about factor of about two when photoionization is turned on, leading to the $20 \%$ increase in the rms emittance near the target seen in Fig. 5. This emittance increase, however, is mainly in the transverse velocity and is not reflected in the spot size. In fact, the beam rms radius at the waist location is about $10 \%$ smaller than the corresponding case without photoionization, as seen in Fig. 6. This seeming inconsistency occurs because the beam enters the photoionized plasma surrounding the target before it undergoes significant photostripping, so the beam first experiences a period of reduced space charge before the later increase. An examination of the net current for these cases shows that the beam is $80-90 \%$ current neutralized near the waist, both with and without photoionization. However, due to the higher charge state, the net current is much higher with photoionization, and the self-magnetic field approaches $15 \mathrm{kG}$ at its maximum. Nonetheless, the self-magnetic field has a negligible effect on the transverse dynamics for these parameters because it builds up only in the immediate vicinity of the target.

Photoionization has also been included in several foot-pulse simulations. In these cases, photoionization only becomes important about $10 \mathrm{~ns}$ after the beam head arrives, due to the time required to heat the hohlraum. The effects of improved charge neutralization are therefore only evident in a $10 \%$ reduction in the tail radius of the foot pulse. Since this part of the pulse is only needed for maintaining the hohlraum temperature neat $100 \mathrm{eV}$, this improvement has little importance. However, the wide-angle distributed-radiator target recently described by Callahan, et al.[27] requires more than half of the foot-pulse energy to be deposited in the final $8 \mathrm{~ns}$, due to the greater mass near the target mid-section. In this case, improved neutralization by a photoionized plasma may significantly improve the transport of this higher-current foot-pulse tail. 

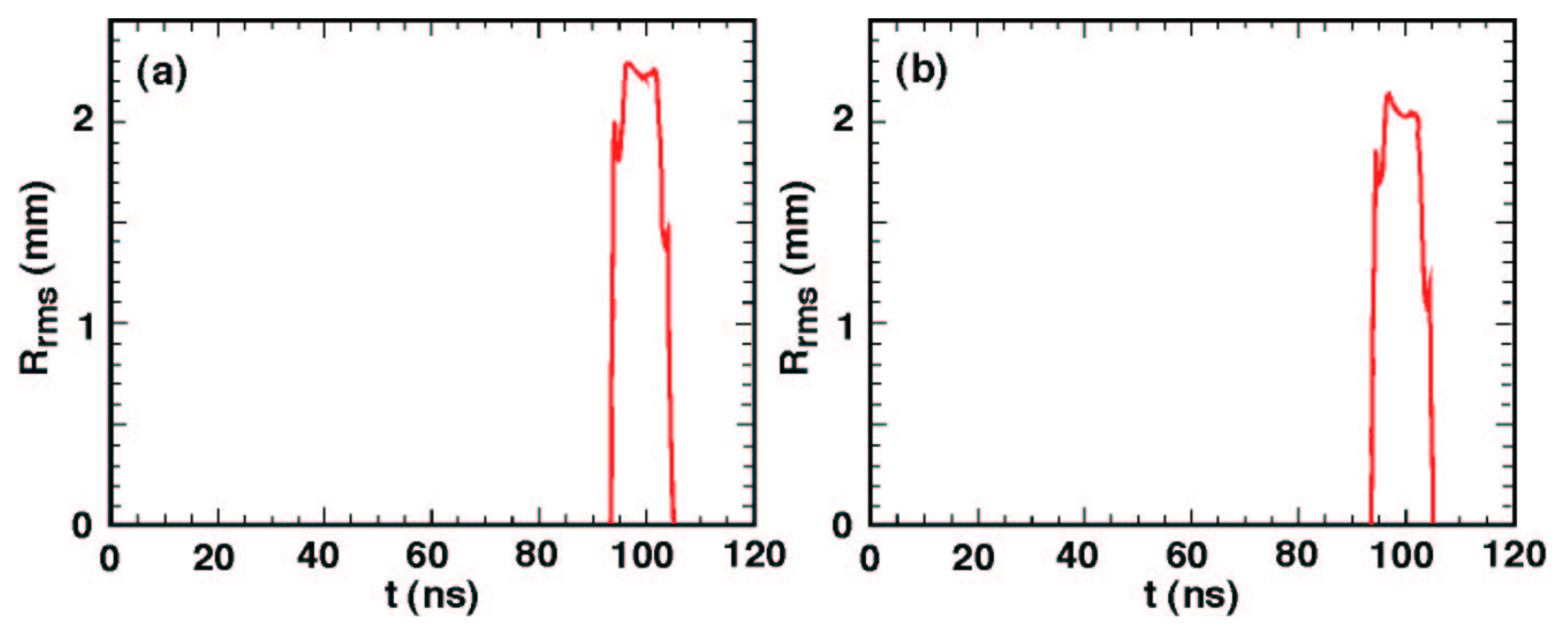

Figure 6: Time variation of the main-pulse rms radius near the waist (a) with and (b) without photoionization for a 15-mrad convergence angle. An expanded verticle scale is used here to highlight differences.

\section{CONCLUSIONS}

The chamber-transport simulations reported here are the first to use parameters approximating those favored for a heavy-ion-fusion driver. The beams have a higher current and lower rigidity than values used in previous simulations, and several previously neglected physical features have been included in the model, such as the 3-m beam ports, multiple ionization of the background gas, and photionization by target X rays. Both main pulses and the "foot" pulses used to heat the hohlraum have calculated focal spots that marginally match the requirements of recent distributed-radiator targets. This good performance is largely due to pre-neutralization by layers of hydrogen plasma near both ends of the beam port. Some improvement in the focal spot results from using a 15 mrad convergence angle instead of the nominal $10 \mathrm{mrad}$ angle, but the larger entrance hole complicates chamber design and neutron shielding. Photoionization further improves the focal spot of main pulses by about $10 \%$ but, as expected, has a minimal effect on foot pulses.

Collaboration with target and accelerator designers is still needed to develop an optimized and integrated scenario for chamber transport. In future work, we will determine the most effective choices for the beam convergence angle, and the size and density of the neutralizing plasma. Work is beginning on the use of more-realistic input beam distributions, including the time-variation of the foot-pulse current, and several additional features of chamber physics will be added to the numerical model, particularly some representation of the molten-salt jets.

\section{Acknowledgments}

This work was performed under the auspices of the US Department of Energy by University of California Lawrence Livermore National Laboratory and Lawrence Berkeley National Laboratory under Contracts No. W-7405-ENG-48 and DE-AC-3-76SF00098.

[1] C. L. Olson, Nucl. Instr. and Meth in Phys. Res. A 464, 118 (2001).

[2] D. A. Callahan, Fusion Eng. and Design 32-33, 441 (1995).

[3] N. Barboza, Fusion Eng. and Design 32-33, 453 (1995).

[4] J.-L. Vay and C. Deutsch, Fusion Eng. and Design 32-33, 467 (1995).

[5] W. M. Sharp, D. A. Callahan-Miller, A. B. Langdon, M. S. Armel, and J.-L. Vay, Nucl. Instr. and Meth in Phys. Res. A 464, 284 (2001).

[6] T. Kikuchi, S. Kawata, S. Kato, S. Hanamori, and M. Yazawa, Jpn. J. Appl. Phys. 38, 270 (1999)

[7] P. C. Efthimion and R. C. Davidson, Nucl. Instr. and Meth in Phys. Res. A 464, 310 (2001). 
[8] W. M. Sharp, D. A. Callahan-Miller, M. Tabak, S. S. Yu, and P. F. Peterson, "Chamber Transport of 'Foot' Pulses for Heavy-Ion Fusion," submitted to Phys. Plasmas.

[9] D. V. Rose, D. R. Welch, B. V. Oliver, W. M. Sharp, and A. Friedman, Nucl. Instr. and Meth in Phys. Res. A 464, 299 (2001).

[10] D. R. Welch, D. V. Rose, B. V. Oliver, T. C. Genoni, and R. E. Clark, Phys. Plasmas 9, 2344 (2002).

[11] T. P. Hughes, R. E. Clark, and S. S. Yu, Phys. Rev ST Accel. Beams 2, 110401 (1999).

[12] D. R. Welch, D. V. Rose, B. V. Oliver, and R. E. Clark, Nucl. Instrum. Meth. Phys. Res A 464, 134 (2001).

[13] G. Lapenta and J. U. Brackbill, J. Comp. Phys. 115, 213 (1994).

[14] R. E. Olson, Nucl. Instr. and Meth in Phys. Res. A 464, 93 (2001).

[15] M. S. Armel, "Atomic Processes for Heavy Ion Inertial Fusion," Ph. D. Thesis, University of California Berkeley (2000).

[16] W. R. Meier, private communication.

[17] W. R. Meier, R. O. Bangerter, and A. Faltens, Nucl. Instrum. Meth. Phys. Res A 415, 249 (1998).

[18] W. R. Meier, J. J. Barnard, and R. O. Bangerter, Nucl. Instrum. Meth. Phys. Res A 464, 433 (2001).

[19] J. D. Lawson, J. Electron. Control 5, 146 (1958).

[20] M. Tabak and D. A. Callahan-Miller, Nucl. Instr. and Meth in Phys. Res. A 415, 75 (1998).

[21] D. A. Callahan-Miller and M. Tabak, Nucl. Fusion 39, 1547 (1999).

[22] R. W. Moir, R. L. Bieri, X. M. Chen, T. J. Dolan, M. A. Hoffman, P. A. House, R. L. Leber, J. D. Lee, Y. T. Lee, J. C. Shrock, M. T. Tobin, and W. H. Williams, Fusion Technol. 25, 5 (1994).

[23] D. R. Olander, G. Fukuda, and C. F. Baes Jr, Fusion Science Technol. 41, 141 (2002).

[24] M. Reiser, Theory and Design of Charged Particle Beams (John WIley \& Sons, New York, 1994), p. 341ff.

[25] A. B. Langdon, R. O. Bangerter, and D. A. Liberman, Nucl. Instrum. Meth. Phys. Res A 278, 68 (1989).

[26] A. B. Langdon, Part. Accel. 37-38, 175 (1992).

[27] D. A. Callahan-Miller, M. C. Herrmann, and M. Tabak, "Progress in Heavy-Ion Target Hohlraum and Capsule Design," to be published in Laser Part. Beams (2002). 
University of California

Lawrence Livermore National Laboratory

Technical Information Department

Livermore, CA 94551

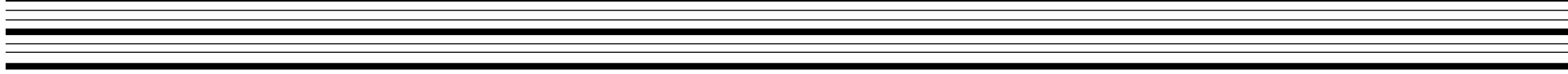

